\title{
Parametric design, fabrication and validation of one-way polymeric valves for artificial sphincters
}

\author{
Tommaso Mazzocchi ${ }^{\mathrm{a}, *}$, Leonardo Ricotti ${ }^{\mathrm{a}}$, Novello Pinzi ${ }^{\mathrm{b}}$, Arianna Menciassi ${ }^{\mathrm{a}}$ \\ a The BioRobotics Institute, Scuola Superiore Sant'Anna, Viale R. Piaggio, 34, 56025, Pontedera, PI, Italy \\ b Dipartimento di Urologia, Università degli Studi di Siena, Via Banchi di Sotto, 55, 53100 Siena, SI, Italy
}

\section{A R T I C L E I N F O}

\section{Article history:}

Received 5 January 2015

Received in revised form 22 June 2015

Accepted 7 July 2015

Available online 15 July 2015

\begin{abstract}
A B S T R A C T
The design of artificial sphincters requires an accurate dimensioning of dedicated valves, normally made of polymeric materials. This effort is also interesting for developing fluid and pressure regulating solutions related to other biomedical and non-biomedical fields. In this article we focused on the parametric design of polymeric valves, by taking inspiration from commercially exploited solutions used in the food industry and performing appropriate scaling in order to make them suitable for artificial organs and components. In addition, different materials with diverse mechanical properties were considered, focusing on a lowcost fabrication approach. Finite element model analyses were conducted to simulate the behavior of different valve profiles and to predict the valve opening pressure. Simulation results were validated by comparing them with experimental results, obtained by fabricating and testing different valve types. This polymeric valve parametric analysis may be exploited for the design of artificial sphincters, having the potential to tackle urinary incontinence, a disease that affects about 350 million people worldwide.
\end{abstract}

(c) 2015 Elsevier B.V. All rights reserved.

\section{Introduction}

Approximately 350 million individuals worldwide experienced urinary incontinence (UI) in 2008 and this number is expected to increase up to 423 million by 2018 [1]. UI is a disorder that produces an involuntary urine loss caused by an uncontrolled overactive bladder or a pelviperineal muscle deficit that can arise as a surgery complication in male subjects (e.g. after prostate surgery), while in female subjects it may occur after childbirth or after urogenital organs surgery. In hospitals, UI diseases are normally managed by using absorbent materials or urethral catheters provided with a bag (tied to the patient's leg). Extraluminal sphincters (ES) are devices able to control fluid flow through an elastic duct, by modulating the compression of the elastic duct wall itself. They need to be implanted via. surgical procedures, so they are rather invasive. The most common ES that are commercially available to tackle UI are the ProAct system (Uromedica, MN, USA) and the AMS 800 (American Medical Systems, Minnetonka, MN, USA). These solutions require a surgical procedure for their installation and they are only available for males [2,3]. ProAct and AMS 800 are expensive and can lead to complications such as erosion, infection and mechani-

\footnotetext{
* Corresponding author. Fax: +39 50883101.

E-mail address: t.mazzocchi@sssup.it (T. Mazzocchi).
}

cal failure. Therefore, periodic revisions (rather complicated to be performed) are mandatory. In addition, if replacement is needed, a second invasive surgical procedure needs to be performed.

On the other hand, intraluminal sphincters (IS) do not require a surgery procedure to be implanted: they are usually installed by using the duct orifice and brought to the site of interest through a pushing catheter. At present, few commercial IS are available, such as the FemSoft (Rochester Medical Corporation, MN, USA) or the InFlow device (Vesiflo, Inc, WA, USA) [4]. IS are not completely internal devices: a small portion of the system is inevitably visible, thus raising psychological discomfort in certain situations.

Recently, advanced and rather complex mechatronic solutions have been proposed to tackle UI. Lamraoui et al. reported the design, development and in vivo testing on goats of a $60 \mathrm{~cm}^{3}$ artificial sphincter (AS) prototype composed of standard mechanical and electrical components [5]. More recently, a retro-compatible device was proposed by Hached and colleagues. The system was based on a hydraulic module and a control unit and showed good efficacy in both bench tests and ex vivo tests [6]. The same authors also recently developed a wirelessly controlled and adaptive AS, capable of postoperative occlusive cuff pressure modification [7]. All the above-mentioned mechatronic solutions are rather invasive and their long-term safety and efficacy still have to be demonstrated. 


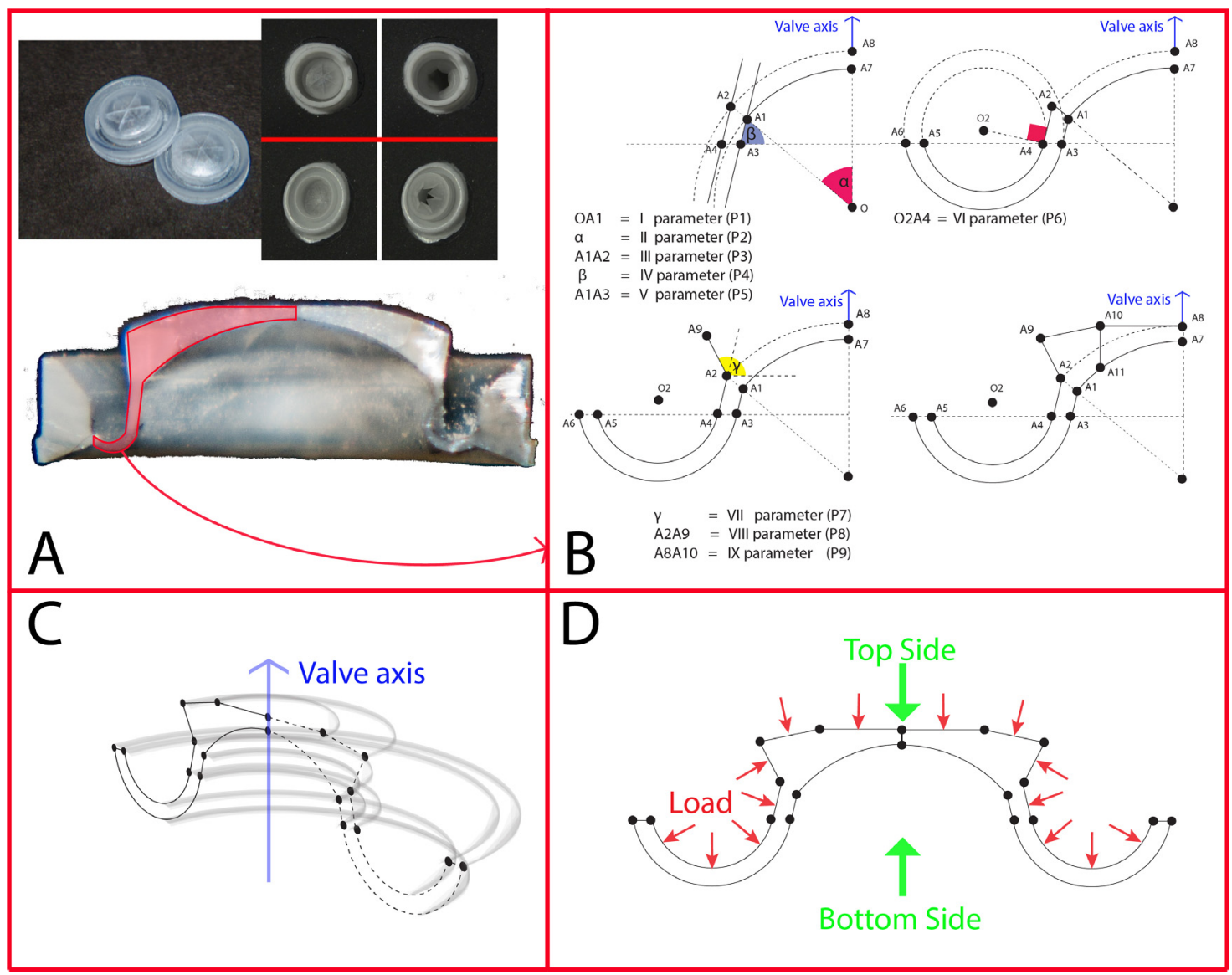

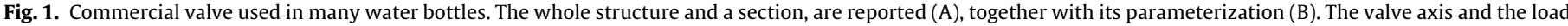
distribution are shown in $C$ and $D$, respectively.

The authors also recently highlighted a possible new design for IS, based on magnetically responsive mechanisms able to engage a unidirectional valve and thus modulate its stiffness [8]. At present, a UI solution suitable for both sexes featuring high stability and low invasiveness (without altering the body scheme) is not available.

Passive unidirectional polymeric valves are a key component of devices designed to tackle UI diseases. They are useful to control the liquid pressure between two compartments, thus ensuring a unidirectional flow when a certain pressure threshold is overcome. Biocompatible polymers are obviously needed and independence from energy sources would reduce the intrinsic hazards related to the use of such systems.

The achievement of an artificial IS suitable for both sexes requires an accurate dimensioning of ad hoc one-way passive valves. To this purpose, the analysis of available solutions for other biomedical or non-biomedical fields can provide some interesting hints. Many literature studies have focused on the correlation between geometry and valve performance, highlighting the importance of numerical simulations such as non-linear finite element models (FEM) in order to optimize the design and the sizing of valve profiles. In the biomedical field, this approach has been extensively used to design heart valves. Different valve profiles have been reviewed by Mohammadi and Mequanint [9], in terms of both modelling and design strategies. Recently, Burriesci and colleagues focused on a novel design strategy for polymeric heart valves (supported by numerical simulations), in order to increase valve performances and reduce stress levels [10]. Another interesting study was carried out by Labrosse et al., which developed a geometric model of an aortic trileaflet valve, for the purpose of evaluating how much the dimensions of the aortic valve components could be varied, while still maintaining proper target performances [11]. More recent studies focused on applying FEM analyses to properly design leaflet materials and to predict the hydrodynamic behavior of nanocomposite-based polymeric aortic valves $[12,13]$. However, the aforementioned studies cannot be easily generalized and applied to the design of a urinary system valve. This is mainly due to the rather complicated design and manufacturing processes, needed to build heart valves which require several steps in order to combine a metallic stent with the polymeric leaflets.

A different approach must be adopted when facing scalable manufacturing in the presence of high pressure ranges and small flow rates $(\mu \mathrm{l} / \mathrm{min})$. Although these conditions are not typical of the urinary system, this approach has been widely adopted for the design of micro-valves to be used in microfluidic systems. The geometrical parameters of the valve, including shape, fluid channel width and membrane thickness are usually examined. Snakenborg et al. designed a polydimethylsiloxane (PDMS) membrane provided with an incision in order to fabricate a check valve able to control the flow rate [14]. Mohan et al. reported interesting considerations concerning the design and the application of elastomeric microvalves, focusing on actuation pressure minimization, reliable operation and convenient integration into complex microfluidic devices [15].Jahanshahi et al. focused on the design of a micro-valve characterized by a high pressure range and a relatively high sealing capability (no flow up to 0.6 MPa reverse pressure) [16]. Hilbert and colleagues introduced an innovative micro-valve manufacturing technique by using PDMS loaded with neodymium particles, to adjust the switching point of each single valve [17].

Hickerson and colleagues developed a valve design strategy suitable for scalable manufacturing, and based on standard tech- 
niques that allow the development of low-cost disposable systems. The reported results may be exploited for many applications that require specific valve performance. However, they are not usable as components for artificial urinary sphincters, due to the high pressure range (12 $\mathrm{kPa}-120 \mathrm{kPa})$ and to the small flow rates [18].

Uohashi and colleagues took into account different one-way valve profiles, achievable by exploiting standard molding processes. They ensured high flow rates $(\mathrm{ml} / \mathrm{min})$. Interesting results, concerning the influence of material stiffness on valve performance, were reported. However, an accurate relationship between valve geometrical parameters and performance was not highlighted [19].

In this article, we discuss the parametric design, fabrication and validation of a one-way polymeric valve as component of AS, taking as a model a common valve profile available in water bottles. Low-cost fabrication techniques and a biocompatible and stiffnesstunable material were considered for valve design and fabrication. Simulation results evidenced the most suitable profiles in correspondence to certain desired opening pressures. For application in the urinary system, hermetic closure should be assured: sudden coughs and involuntary abdominal muscle contractions can generate pressures up to $12 \mathrm{kPa}$. However, this physiological parameter depends on the patient's anatomical features, therefore a tunable opening pressure (OP) is desirable [20-22]. As highlighted below, this can be achieved by making small changes to the valve profile geometry or by slightly changing material stiffness.

\section{Experimental}

\subsection{Valve profile and its parameterization}

To select the valve profile, we took inspiration from the food industry, especially from the valves integrated in many water bottles. Normally, they are used to guarantee hermetic sealing up to a certain pressure, a feature that makes it easier to drink liquids during sport activities, for example. We considered a commercial valve that had a vault shape and a surface featuring a six-pointed star die cut. The notches allow the valve to open when the pressure applied on the top surface is above a certain threshold. We analyzed the valve profile and extracted 9 parameters able to describe it, as shown in Fig. 1.

The nine parameters that allow reproduction of the commercial valve were: $\mathrm{P} 1_{\mathrm{c}}=3.7 \mathrm{~mm}, \mathrm{P} 2_{\mathrm{c}}=50^{\circ}, \mathrm{P} 3_{\mathrm{c}}=0.20 \mathrm{~mm}$, $\mathrm{P} 4_{\mathrm{c}}=83^{\circ}, \mathrm{P} 5_{\mathrm{c}}=1.28 \mathrm{~mm}, \mathrm{P} 6_{\mathrm{c}}=0.37 \mathrm{~mm}, \mathrm{P} 7_{\mathrm{C}}=97^{\circ}, \mathrm{P} 8_{\mathrm{c}}=1.00 \mathrm{~mm}$ and $\mathrm{P} 9_{\mathrm{C}}=1.70 \mathrm{~mm}$ (where $\mathrm{C}=$ commercial).

We aimed at fabricating one-way valves with a specific OP, by taking into account the fabrication constraints. The analysis of the nine parameters led to a redundant problem. However, all parameters were varied, in order to highlight how their different values influenced overall valve performance. For each parameter, five equidistant values were identified (Table 1). Moreover, they were combined with six different types of material stiffness.

The first analysis aimed at assessing how valve performance changed when applying an isotropic scaling (increasing or decreasing valve size by a scale factor that was the same in all directions). To this aim, P1 was considered because it is related to the radius of the vault valve. A valve radius suitable for urological applications must be smaller than $6 \mathrm{~mm}$ [23]. The scaling factors (SF) analyzed in this work were $0.35,0.67,1.00,1.32$ and 1.64 . They corresponded to P1 values of $1.3,2.5,3.7,4.9$ and $6.1 \mathrm{~mm}$, respectively. Differently scaled valves may find applications in different fields.

Another important aspect of the analysis concerned how valve performance changed in function of the other eight parameters, once a certain valve size was selected. This allowed us to identify the most relevant parameters for valve performance.

\subsection{Finite Element Model (FEM) simulations}

Matlab ${ }^{\circledR}$ R2013a (MathWorks) and Abaqus 6.13 (Dassault Systemes) were used to manage data and to perform FEM simulations, respectively.

Matlab $^{\circledR}$ environment was used to manage the different parameters. Once the desired valve profile was generated, Matlab ${ }^{\circledR}$ generated an appropriate file, readable by Abaqus, which loaded the desired valve geometry, for detecting the OP of the respective profile. This allowed us to handle a large number of simulations in a simple manner. The valve model was implemented using Python language.

When Abaqus had processed the simulation, it generated a file in which all the results were reported. An algorithm was developed to compare each simulation and to identify the valve OP. The algorithm tracked all the extreme points (A8 and A7 in Fig. 1) for each valve strip: when the distance between such extreme points was double than the valve thickness (P3), the valve was considered open. Once the simulation was completed, the extreme points of the valve strip reached during the simulation were stored in a file, from which Matlab ${ }^{\circledR}$ was able to indentify the valve OP.

After creating the valve in the Abaqus environment, experimental data (stress-strain curves) of the material used were imported by exploiting the Hyperplastic module supported by the program. In the Abaqus Step module, an explicit dynamic behavior was defined, by imposing a mass scaling equal to 100 . Appropriate tie constraints between the described elements were implemented, thus reproducing the commercial valve of Fig. 1A.

The valve model was provided with a fixed constraint as boundary condition, applied on the surface obtained from the segment (A5A6) revolution around the valve (A7A8) axis. The top surface of the valve was loaded with a pressure (normal to the valve surface) which linearly increased, until reaching the cracking pressure.

The valve design was composed of three elements. The first one represented the valve basement, obtained from the revolution $\left(360^{\circ}\right)$ around the valve axis of the area delimited by segments A6A5, A5A4, A4A2, A2A1, A1A3 and A3A6 (Fig. 1B). The second valve element was obtained from the complete revolution $\left(360^{\circ}\right)$ around the valve axis of the area delimited by sections A1A2, A2A9, A9A10 and $A 10 A 11$. The third element referred to the valve strips obtained when the die cut was applied on the top surface of the polymeric valve. A single strip was obtained from a revolution of $60^{\circ}$ around the valve axis of the area delimited by sections A10A8, A8A7, A7A11 and A11A10.

The first element (valve basement) was meshed with 2160 cells, the second (valve head) was meshed with 1224 cells. The third element (valve strip) was meshed with 81 cells. A structured Hex mesh was used for the three bodies. To demonstrate that the mesh was suitable for the target application, we carried out mesh refinement tests (Supplementary materials, Table S1). The mentioned mesh size value was identified as a good compromise between adherence of the predicted OP values to the real ones and the time needed to complete each simulation. Once the mesh grid of the valve model was defined, a set of nodes was generated in order to store the segment (A8A7) displacement values for each valve strip, during the entire simulation. Therefore, 12 mesh nodes were selected to detect valve opening. Von Mises stresses were also recorded for each simulation.

\subsection{Polymer synthesis and mechanical testing}

The material selected for valve fabrication was PDMS (Sylgard 184, Dow Corning). This material is particularly suitable for biomedical applications, due to its high biocompatibility, its tunable mechanical properties, and its good thermal and chemical stability. Furthermore, PDMS surface can be activated by oxy- 
Table 1

Parameter values considered during FEM simulations.

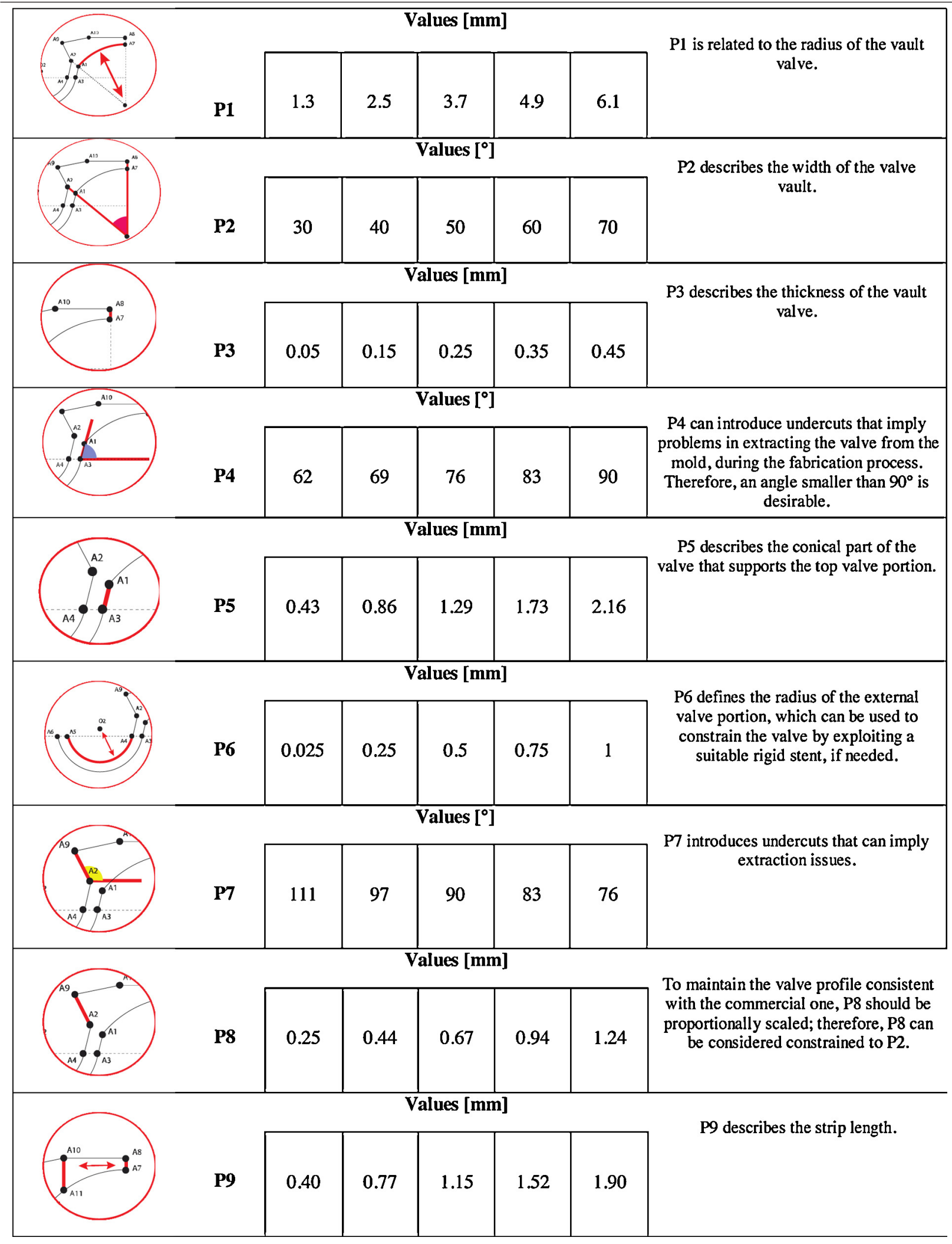

gen plasma, for modifying surface properties and mechanical and chemical performances [24],[25].

PDMS stiffness can be modulated by varying the ratio between the monomer and its curing agent. In this work, 6 different monomer/curing agent ratios were analyzed: 5:1, 10:1, 20:1, 30:1, 40:1 and 50:1 (w/w)

Sample mechanical properties were evaluated with an INSTRON 4464 Mechanical Testing System, by using a $\pm 10 \mathrm{~N}$ load cell, and 
performing traction tests. Samples were cut into $20 \times 5 \times 2 \mathrm{~mm}^{3}$ slices and allocated between two aluminium clamps. All samples were pulled at the constant speed of $5 \mathrm{~mm} / \mathrm{min}$, until reaching sample failure. Data were recorded at a frequency of $100 \mathrm{~Hz}$, the stress was calculated as the load divided by the cross-section area of tensile specimens, while the strain was calculated as the ratio between the extension and the initial length of tensile specimens. The tensile modulus for each tested sample was calculated starting from the stress/strain curve, according to a standard procedure [26]. Mechanical tests were repeated on three different samples, for each sample type.

\subsection{Valve fabrication}

In order to validate the simulation results, polymeric valves were fabricated by exploiting dedicated molds. The molds were composed of two parts, provided with through holes, needed for aligning the parts and closing the mold (using M3 screws and nuts). To achieve the die cut on the valve surface, a proper frame (designed to constrain the polymeric valve) and a custom blade were used.

Both cutting frame and molds were fabricated by using a 3D printer (HD3000, ProJet). The custom blade was obtained by modifying the cutting edge of an off-the-shelf surgical blade (CHIMO, size 22).

The valve fabrication process was based on the following steps: (1) PDMS preparation: monomer and curing agent were mixed with the desired ratio, then the mixture was degassed for $20 \mathrm{~min}$ in a vacuum chamber. (2) Mold surface treatment: a thin teflon film (LOCTITE 8192) was deposited by spraying it on the mold surface, to facilitate subsequent PDMS removal from the mold. (3) PDMS casting: once the mixture was well degassed, it was casted into the teflon-coated mold. (4) Polymerization: after PDMS casting, the mold was closed and thermally treated at $90^{\circ} \mathrm{C}$ for $3 \mathrm{~h}$, to allow full valve polymerization. (5) Valve extraction and cutting: the mold was opened and the polymeric valve removed. It was then subjected to die cutting by the dedicated frame and custom blade.

\subsection{Valve testing}

To test the polymeric valve functionality, a dedicated experimental set-up was developed (Fig. 2).

The set-up consisted of a motorized piston provided with a fine control of both horizontal stroke and speed. The horizontal stroke kept constant the height of the water column downstream of the valve during the tests, which was needed to detect the valve OP. Speed control was compiled into a development board (STM32F407, ST) exploiting Simulink (MathWorks) environment with a sampling frequency of $1 \mathrm{kHz}$.

To monitor the OP, the development board was connected to a pressure sensor (HCX001D6 V, purchased from Sensor Technics) for wet environments, capable of measuring pressures up to $100 \mathrm{kPa}$.

The mechanical parts of the testing set-up included a Screw Ball (BSSC1510-300-SC10, diameter $15 \mathrm{~mm}$, stride: $10 \mathrm{~mm}$, stroke: $300 \mathrm{~mm}$ ) and appropriate tools for supporting it (BSW12, BUN12, BNFB1505C-30 produced by MISUMI company). These components were assembled on a derlin-polycarbonate frame. A DC-micromotor (Series 2342 012CR, Faulhaber) provided with a Planetary Gearhead to increase the motor torque with a reduction ratio of 43:1 (Series 30/1S3, Faulhaber), a high performance, low-cost, three-channel optical incremental encoder (HEDS-5540, Avago Technologies) and a full H-Bridge (L298, ST) were mounted on the system.

The testing set-up allowed a syringe $(5 \mathrm{ml})$ and its plunger, connected with the piston pusher, to be mounted. A three-way T-joint
Table 2

shows the different PDMS monomer/curing agent ratios and the corresponding Young's moduli, obtained by way of linear fitting of the stress-strain curves in the initial (linear) region.

\begin{tabular}{ll}
\hline Monomer/curing agent ratio & Young's modulus [MPa] \\
\hline $5: 1$ & $4.95 \pm 0.33$ \\
$10: 1$ & $3.55 \pm 0.35$ \\
$20: 1$ & $1.26 \pm 0.16$ \\
$30: 1$ & $0.41 \pm 0.06$ \\
$40: 1$ & $0.14 \pm 0.02$ \\
$50: 1$ & $0.08 \pm 0.01$ \\
\hline
\end{tabular}

connected the valve seat, the pressure sensor and the syringe output. The valve opening direction was horizontal, to avoid gravity effects.

Six polymeric valve types (four valves for each type) were fabricated by using three different molds (thus, three different geometries) and two different material types (10:1 and 20:1 PDMS). The first valve geometry to be fabricated featured a P1 value of $3.7 \mathrm{~mm}$ and a P3 value of $0.2 \mathrm{~mm}$. The second valve geometry featured a P1 value of $2.5 \mathrm{~mm}$ and a P3 value of $0.25 \mathrm{~mm}$. The third valve geometry featured a P1 value of $2.5 \mathrm{~mm}$ and a P3 value of $0.3 \mathrm{~mm}$. The other parameters, common for the different valve geometries, were: $\mathrm{P} 2=50^{\circ}, \mathrm{P} 4=83^{\circ}, \mathrm{P} 5=0.86 \mathrm{~mm}, \mathrm{P} 6=0.25 \mathrm{~mm}$, $\mathrm{P} 7=97^{\circ}, \mathrm{P} 8=0.67 \mathrm{~mm}$ and $\mathrm{P9}=0.13 \mathrm{~mm}$.

Once the twenty-four polymeric valves had been fabricated, they were tested in order to measure their OP (detected using the dedicated sensor to measure pressure drop). The syringe was filled with air or distilled water $\left(\mathrm{d}-\mathrm{H}_{2} \mathrm{O}\right)$ and the piston speed was set at $5 \mathrm{~mm} / \mathrm{s}$. Each valve was tested three times, and six cycles of opening/closure were performed, within each test. Thus, eighteen OP values were recorded for each tested valve.

An additional test was carried out, in order to evaluate the tightness of the fabricated valves. During this test, the piston speed was set at $0.25 \mathrm{~mm} / \mathrm{s}$ and it was stopped before reaching the valve OP. Different constant pressure values were kept for $10 \mathrm{~s}$. This allowed the valve pressure loss to be measured when relatively high pressures were applied and kept constant.

\section{Results and discussion}

\subsection{PDMS mechanical properties}

The stress-strain curves experimentally obtained for the PDMS samples with different mechanical properties as shown in Fig. 3. These curves were imported in the Abaqus environment, in order to properly simulate material mechanical behavior.

Table 2 Young's moduli of the PDMS samples tested, for different monomer/curing agent ratios. Three samples were tested, for each sample type.

\subsection{Simulation results}

Fig. 4 shows some frames captured from valve FEM simulations, in which progressive valve vault compression and opening phases are reported. A dynamic simulation of valve opening and a comparison between the real and the simulated valve are shown in Video S1 (see Supplementary materials).

The analysis of the 9 parameters generated 9 datasets. In each dataset, the 5 parameter values of Table 1 were combined with 6 material stiffness values, thus generating 30 simulation results. A simple approach that immediately highlights macroscopic differences between the obtained datasets is desirable. We fit the simulation results and compared the coefficients obtained from the fitting, in order to identify which parameter was more relevant for 


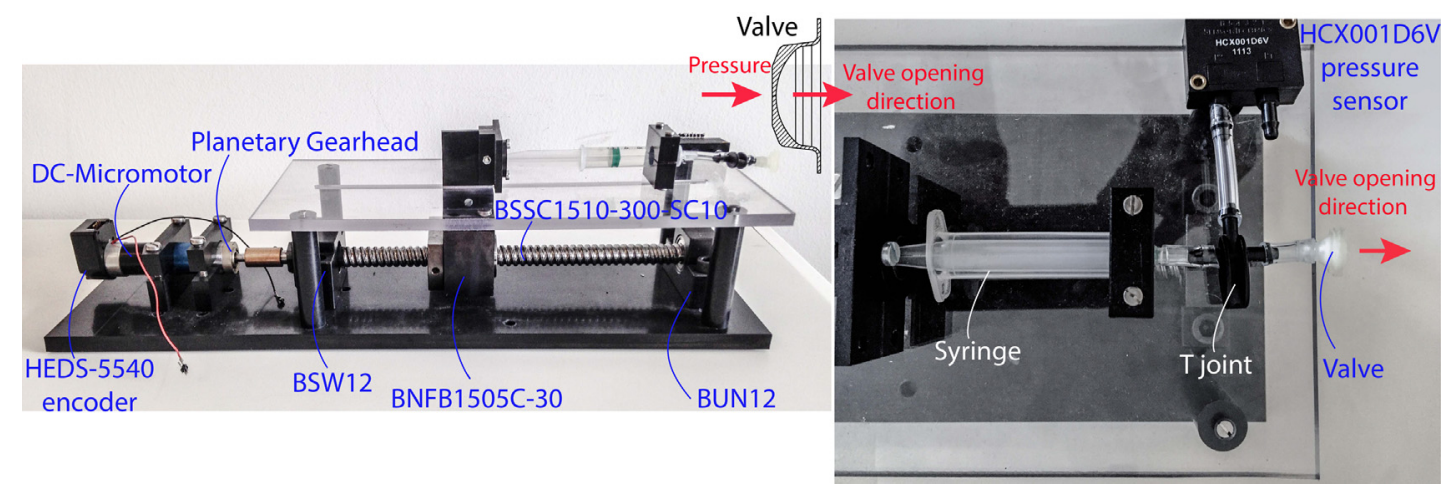

Fig. 2. Valve testing set-up and its components.
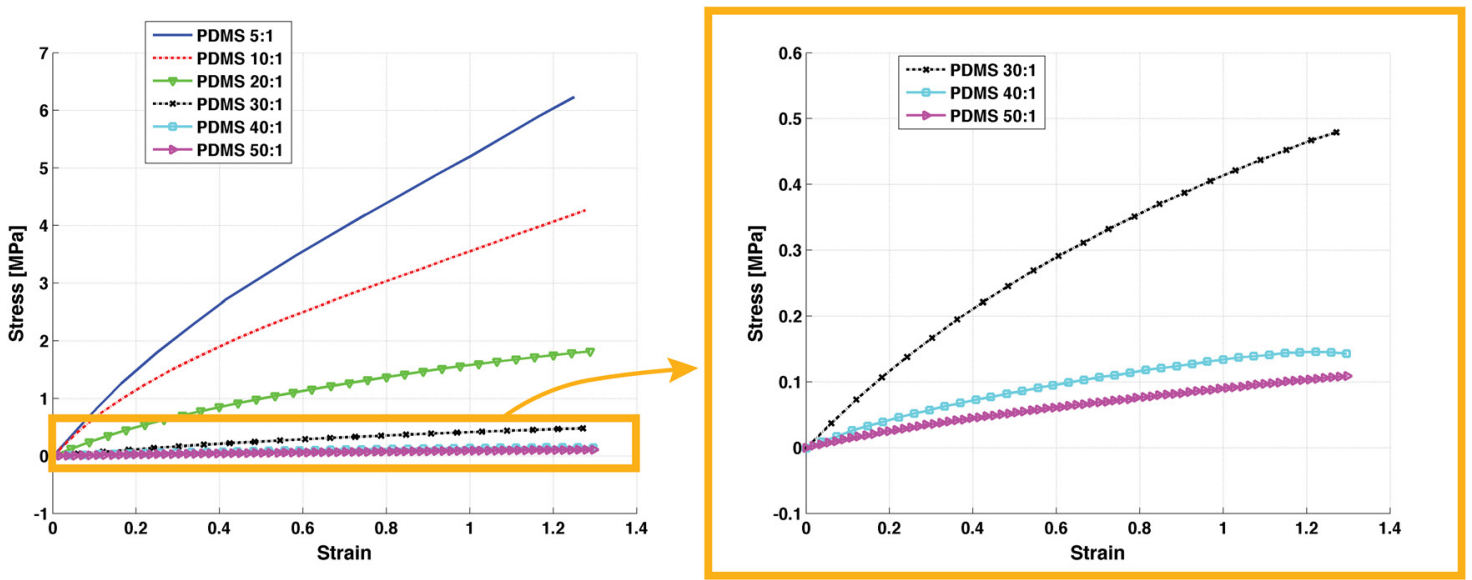

Fig. 3. Representative stress-strain curves of PDMS samples showing different stiffness.
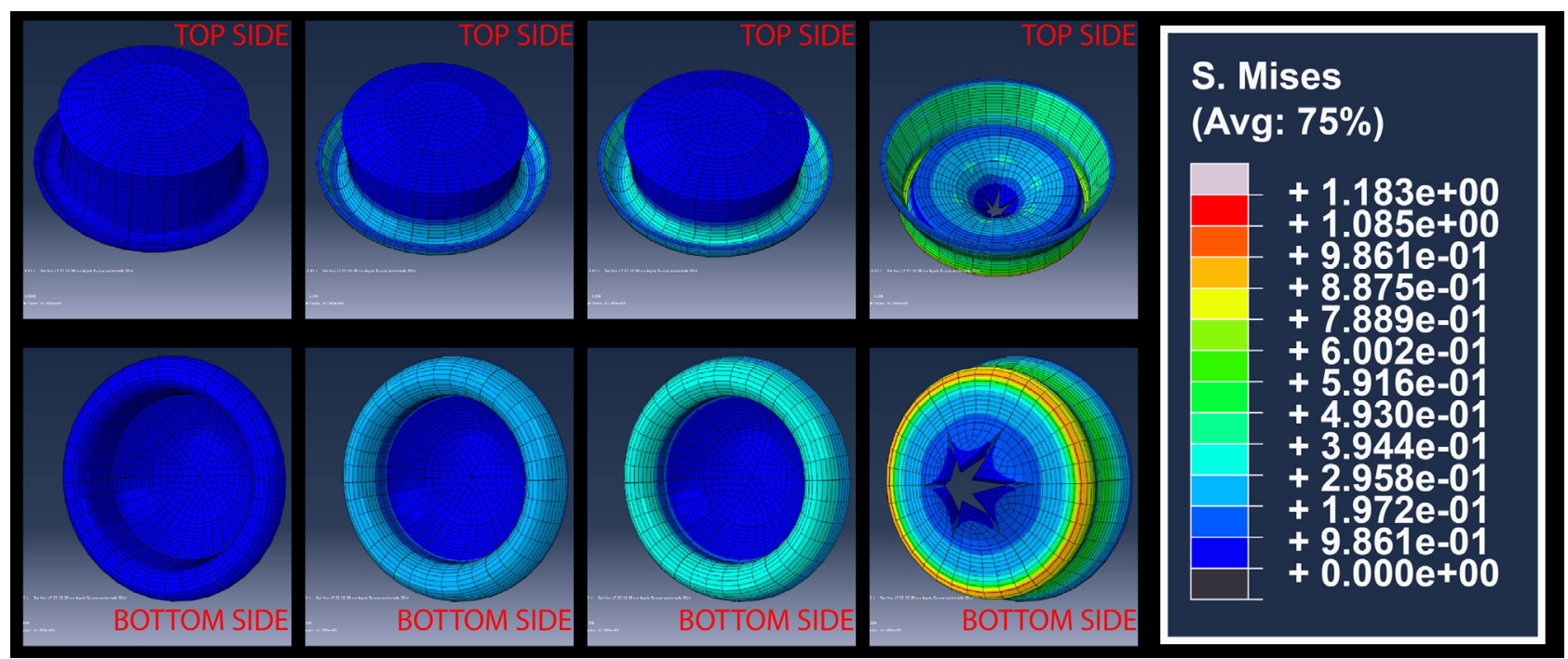

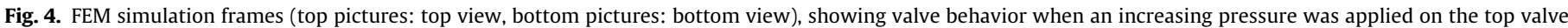
surface. Different colors stand for different von Mises stress values.

the valve performance. The mathematical expression used for data fitting was an exponential function:

$P(\mathrm{x}, \mathrm{y})=\mathrm{a} \times \mathrm{x}^{\mathrm{b}} \times \mathrm{y}^{\mathrm{c}}$

where $\mathrm{x}$ was the material stiffness and $\mathrm{y}$ was the parameter to be evaluated (from P1 to P9). The coefficients a, b and c were obtained by using cftool (Matlab toolbox), and fitting the opening pressure $P(\mathrm{x}, \mathrm{y})$, obtained from FEM simulations, as a function of the mate- rial stiffness and the parameter analyzed. The results are shown in Table 3.

By focusing on the obtained coefficient values, especially with regard to the exponential coefficient $\mathrm{c}$, an immediate comparison between parameters ( $\mathrm{P} 1$ and $\mathrm{P} 9$ ) is possible. The valve thickness (P3) is the most sensitive parameter, being characterized by a $c$ value of 1.6920 . Another particularly sensitive parameter is P4 ( $c$ value: 0.4127 ), which describes the tilt of the top valve portion. As 

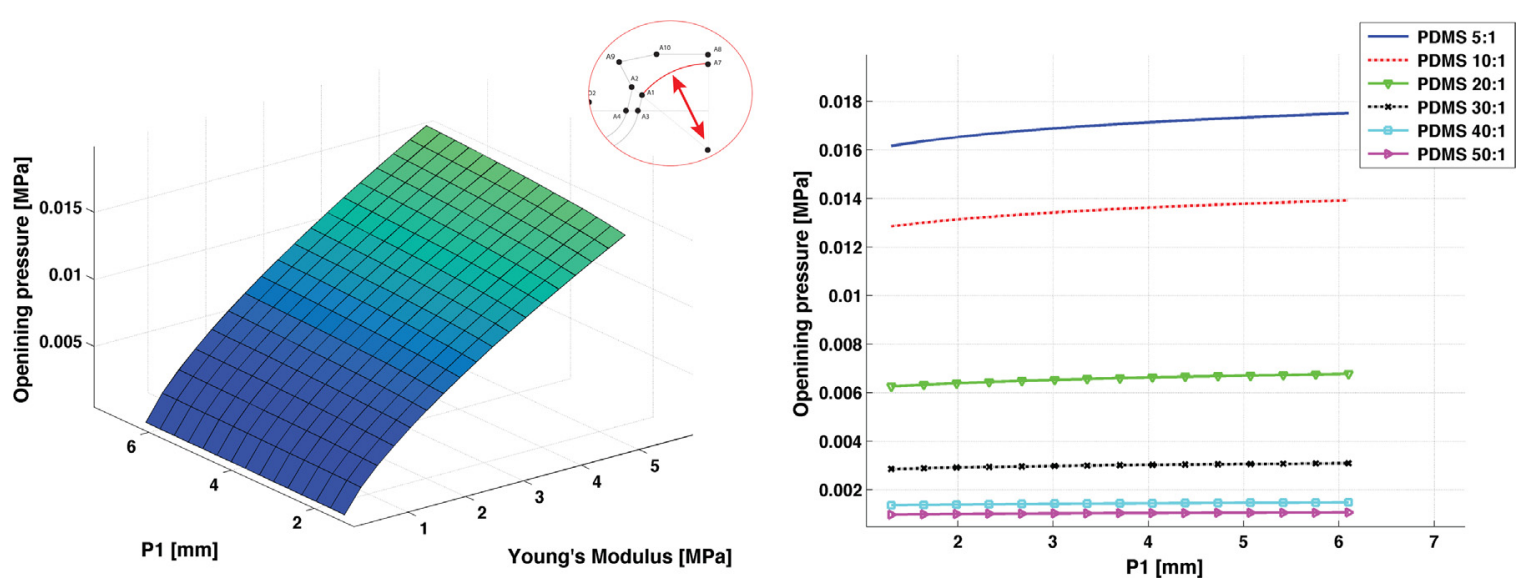

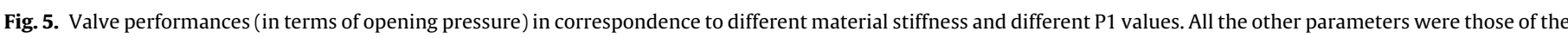

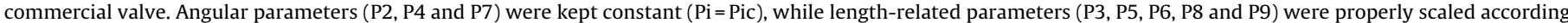
to the homothetic transformation $(\mathrm{Pi}=\mathrm{Pic} \times \mathrm{SF})$.
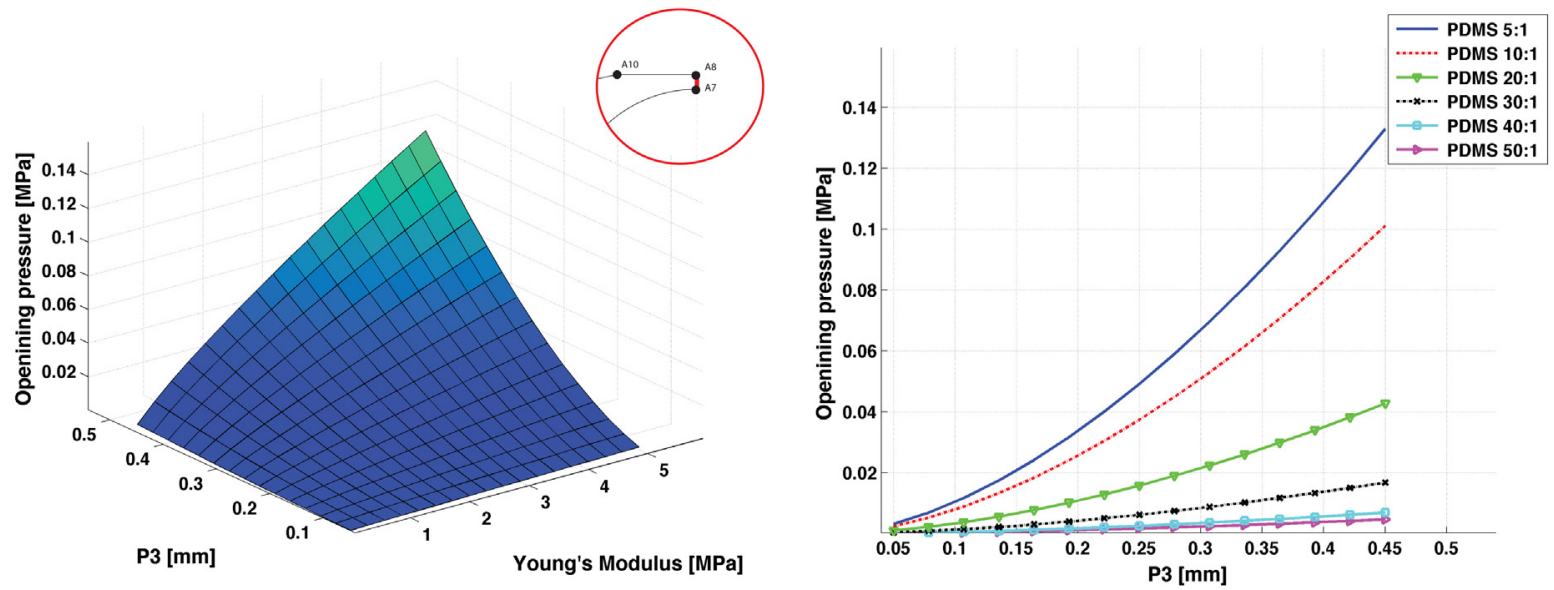

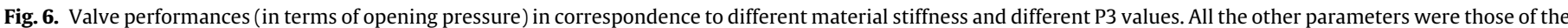

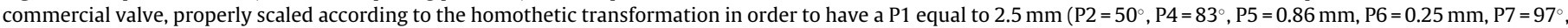
$\mathrm{P} 8=0.67 \mathrm{~mm}$ and $\mathrm{P} 9=0.13 \mathrm{~mm}$ ).

previously mentioned, the design of a polymeric valve showing a specific OP is a redundant task. Therefore, it can be useful to select a design protocol aiming at reducing the overall simulation effort. By varying valve thickness and material stiffness, a large range of OP values is achievable. An efficient design protocol may include: (1) the definition of the valve size and the identification of a suitable scaling factor; (2) the tuning of valve thickness and/or material stiffness. All the other parameters (P2, P5, P6, P7, P8 and P9) play a minor role, as highlighted by the small coefficient values reported in Table 3.

Fig. 5 shows the simulation results obtained by applying a homothetic transformation (i.e. by varying P1). Fig. 6 shows the valve performances when valve thickness (P3) varied.

As mentioned, the other parameters are not crucial for determining valve behaviour. In any case, the correspondent simulation results are reported in Fig. 7.

Table 3

Coefficients $a, b$ and $c$ deriving from the fitting of FEM data, by using Eq. (1).

\begin{tabular}{|c|c|c|c|c|}
\hline \multirow[t]{2}{*}{ Parameter analyzed } & \multicolumn{3}{|c|}{ Coefficient values derived from data fitting } & \multirow[t]{2}{*}{$R^{2}$ value } \\
\hline & $a$ & $b$ & $c$ & \\
\hline P1 & 0.0052 & 0.6956 & 0.0517 & 0.9858 \\
\hline P2 & 0.0055 & 0.7273 & 0.0114 & 0.9898 \\
\hline P3 & 0.1363 & 0.8298 & 1.6920 & 0.9961 \\
\hline P4 & 0.0009 & 0.6991 & 0.4127 & 0.9878 \\
\hline P5 & 0.00786 & 0.7716 & -0.1892 & 0.9926 \\
\hline P6 & 0.0050 & 0.7319 & -0.0183 & 0.9887 \\
\hline P7 & 0.0043 & 0.7481 & 0.0471 & 0.9942 \\
\hline P8 & 0.0057 & 0.7274 & 0.0057 & 0.9890 \\
\hline P9 & 0.0054 & 0.7039 & -0.0052 & 0.9871 \\
\hline
\end{tabular}



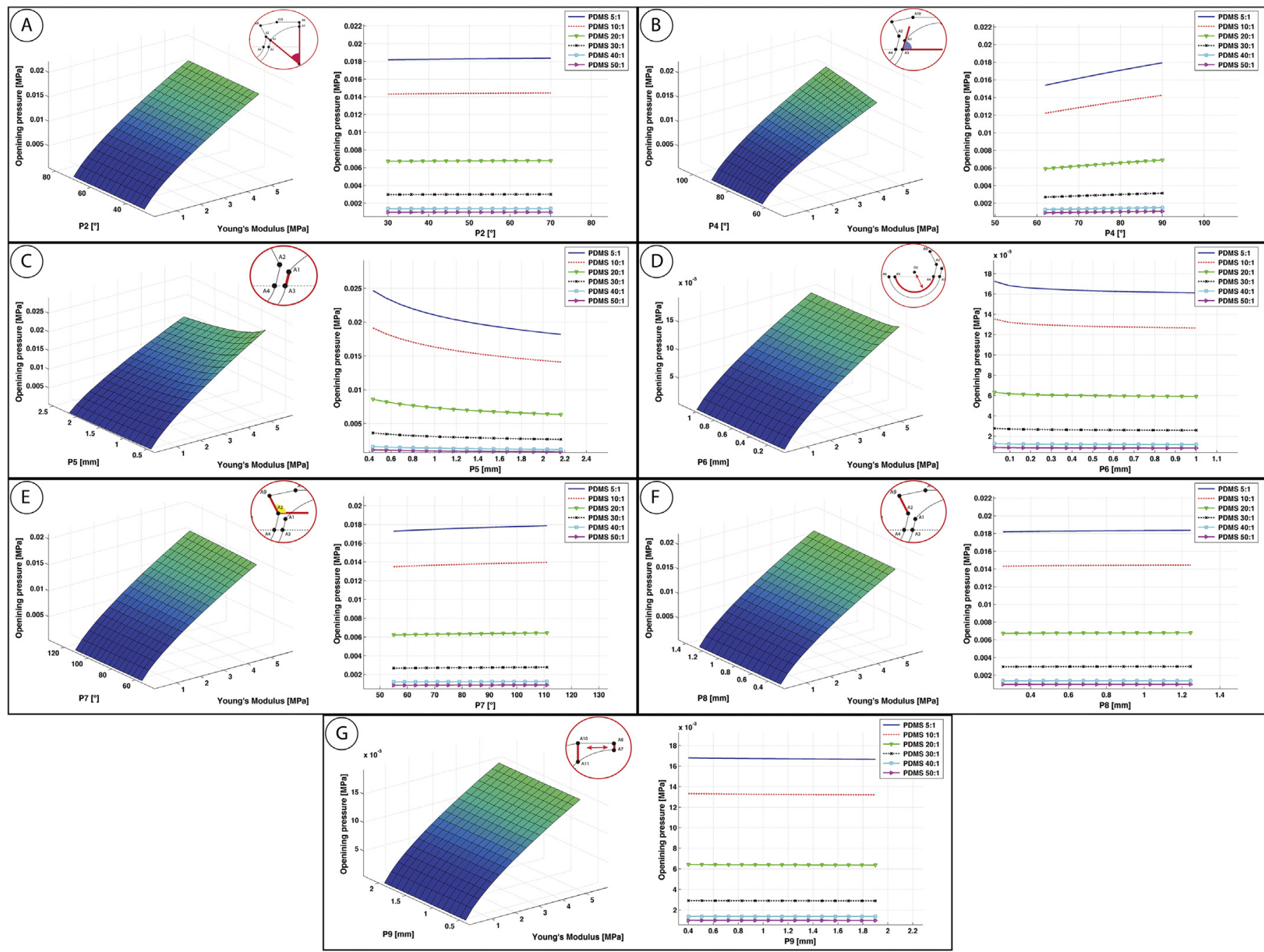

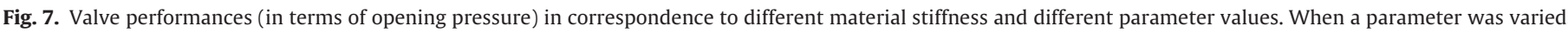

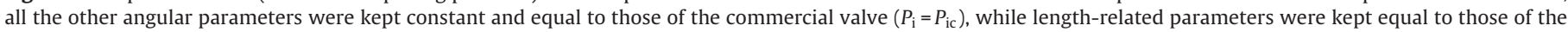
commercial valve, properly scaled according to the homothetic transformation that allowed $\mathrm{P} 1=2.5 \mathrm{~mm}\left(\mathrm{P}_{\mathrm{i}}=\mathrm{P}_{\mathrm{ic}} \times 0.67\right)$.

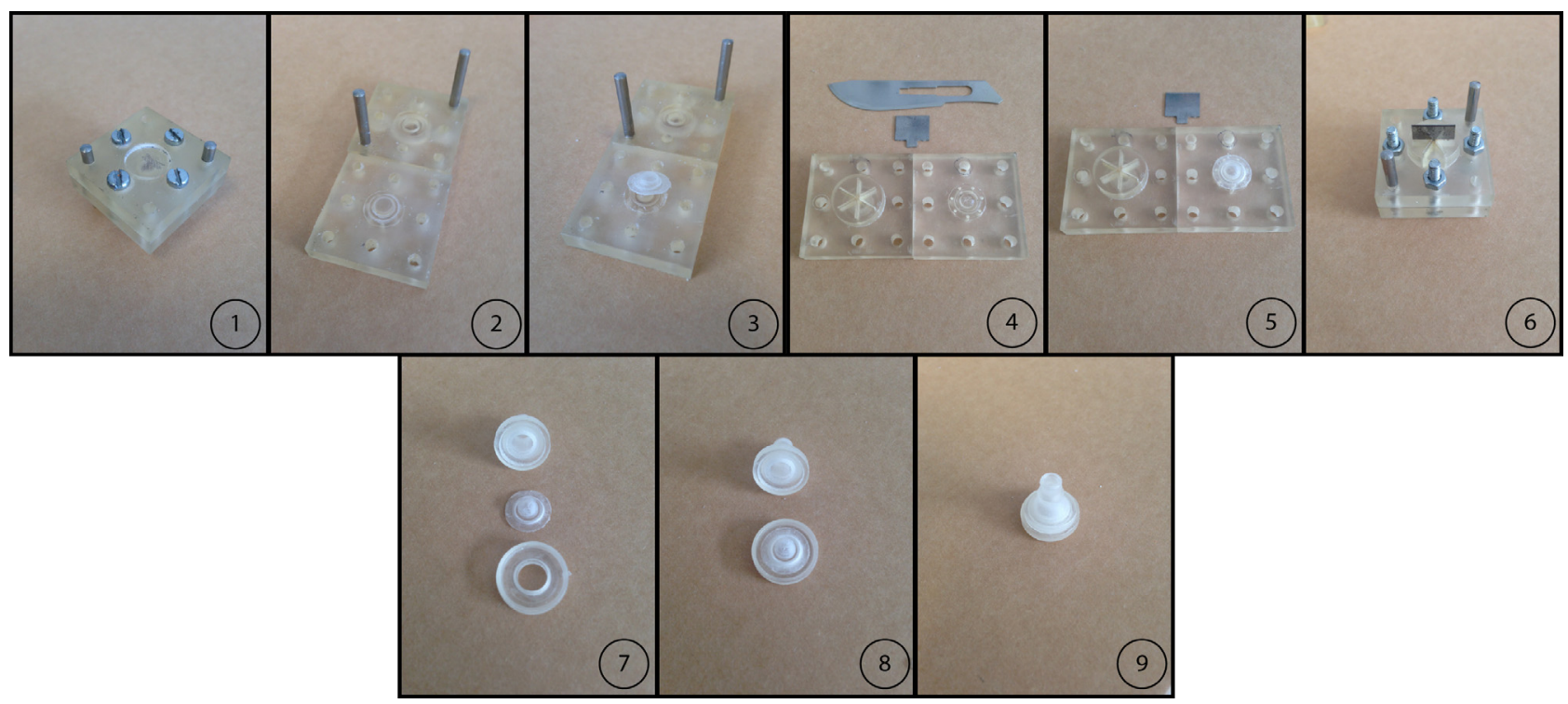

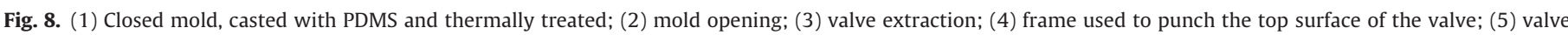

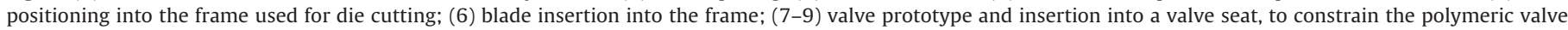
within the testing set-up. 

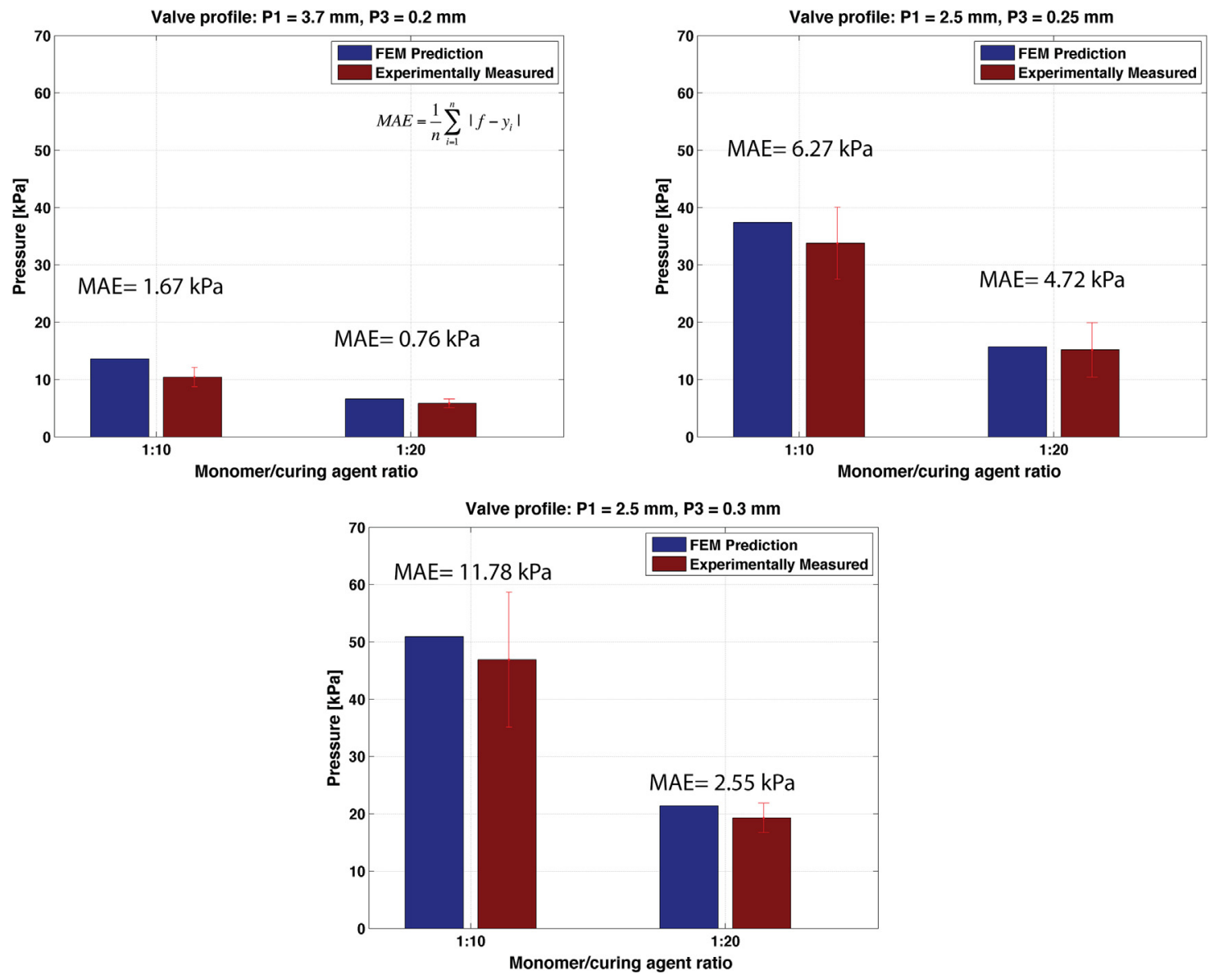

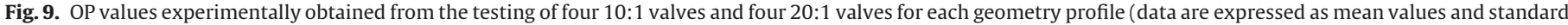
deviations) and comparison with FEM predictions. The mean absolute error (MEA) values are reported for each sample type.

\subsection{Fabrication and validation}

Fig. 8 shows a polymeric valve obtained by using the dedicated mold and cutting frame.

The valve profiles selected to validate the simulations had the following parameters in common: $\mathrm{P} 2=50^{\circ}, \mathrm{P} 4=83^{\circ}, \mathrm{P} 5=0.86 \mathrm{~mm}$, $\mathrm{P} 6=0.25 \mathrm{~mm}, \mathrm{P} 7=97^{\circ}, \mathrm{P} 8=0.67 \mathrm{~mm}$ and $\mathrm{P} 9=0.13 \mathrm{~mm}$. The first tested valve geometry featured $\mathrm{P} 1=3.7 \mathrm{~mm}$ and $\mathrm{P} 3=0.2 \mathrm{~mm}$. The second tested valve geometry featured $\mathrm{P} 1=2.5 \mathrm{~mm}$ (suitable for urinary systems) and P3 $=0.25 \mathrm{~mm}$. The third tested valve geometry featured $\mathrm{P} 1=2.5 \mathrm{~mm}$ and $\mathrm{P} 3=0.3 \mathrm{~mm}$.

For each valve geometry, four PDMS valves made of 10:1 PDMS and four valves made of 20:1 PDMS were fabricated and tested by using the set-up of Fig. 2.

The target OPs can be extrapolated from Eq. (1). FEM simulations predicted that the first valve geometry should have an OP of $13.6 \mathrm{kPa}$ if made of 10:1 PDMS and an OP of $6.6 \mathrm{kPa}$ if made of 20:1 PDMS. The second valve geometry should have an OP of $37.4 \mathrm{kPa}$ if
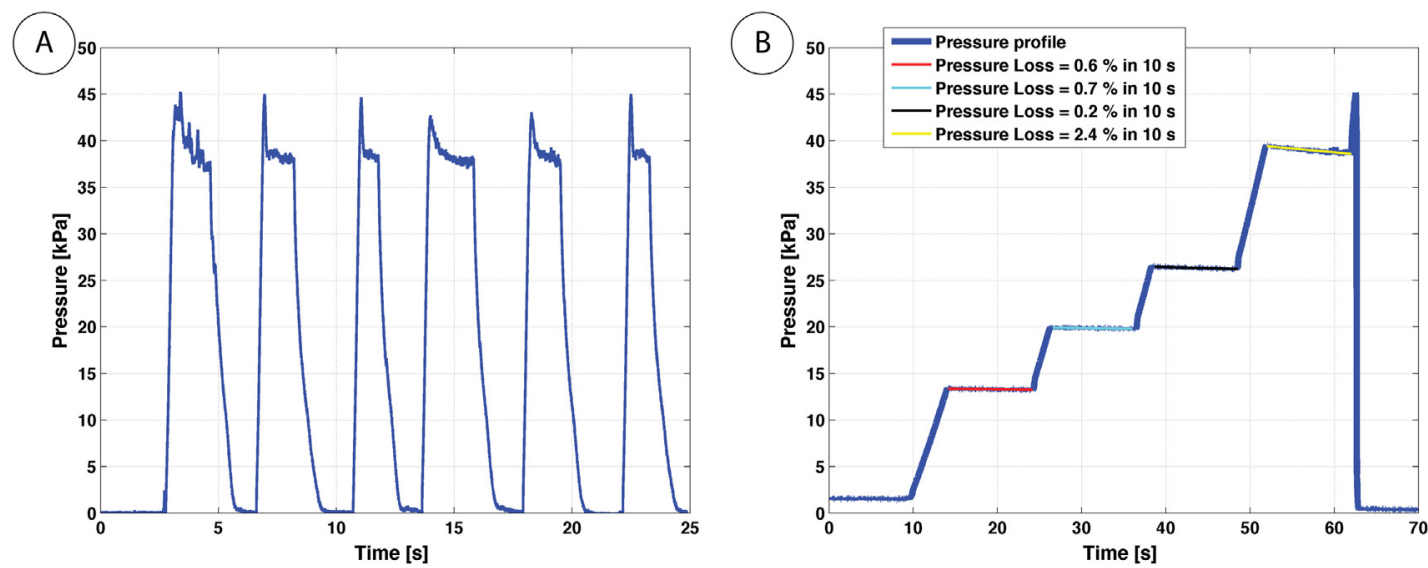

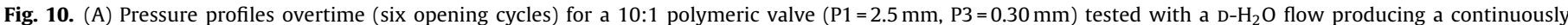

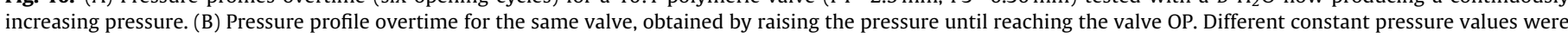
kept for $10 \mathrm{~s}$. These tests allowed us to evaluate valve leakage. 
made of 10:1 PDMS and an OP of $15.7 \mathrm{kPa}$ if made of 20:1 PDMS. The third valve geometry should have an OP of $50.9 \mathrm{kPa}$ if made of 10:1 PDMS and an OP of $21.4 \mathrm{kPa}$ if made of 20:1 PDMS.

In agreement with the conditions set in the simulations, the fabricated valves were solicited by a homogeneous pressure normal to the valve surface. This was achieved by loading them with a liquid flow. The piston advanced with a constant speed of $5 \mathrm{~mm} / \mathrm{s}$ and the pressure detected by the pressure sensor increased until the opening pressure was reached. Afterwards, the pressure immediately fell. The actual performances of the fabricated valves are reported in Fig. 9.

The results obtained demonstrate that the performances of the fabricated unidirectional valves, in terms of OP, are in good agreement with the predictions derived from FEM simulations, with a maximum error of $23 \%$. This allows us to use Eq. (1) as an efficient tool for the design of unidirectional valves for different applications, by playing on the geometric and mechanical features. Concerning the design of an AS capable of tackling UI diseases, the second valve geometry tested $(\mathrm{P} 1=2.5 \mathrm{~mm}$ and $\mathrm{P} 3=0.25 \mathrm{~mm})$ and a 20:1 PDMS monomer/curing agent ratio seem particularly suitable.

Fig. 10A shows pressure loading/unloading cycles performed on a valve prototype. Fig. 10B demonstrates that the valve shows a good tightness: the valve was loaded with relatively high pressure values, kept constant on the valve for $10 \mathrm{~s}$, with small pressure losses. For pressure values up to about half of the valve OP, almost no losses were detected (max pressure loss: $0.7 \%$ in $10 \mathrm{~s}$ ). For higher pressure values, up to $90 \%$ of valve OP, the pressure loss increased, but it did not overcome in any case $2.4 \%$ in $10 \mathrm{~s}$. This further demonstrates the suitability of this technological solution for the development of an AS, as well as for other applications.

This research work can be considered worthy of attention for different reasons. The main purpose of this article is to lay the groundwork for the design and manufacturing of an AS that can tackle UI [27] (see Supplementary materials, Fig. S1). The results reported in this paper permit the design of a polymeric valve showing specific performances in terms of OP. The valve profile has been described by univocally defining the correspondent parameters (these details, in many articles, are partial, omitted, or described in a non parametric manner) $[9,10,16,18,19]$. Another important novelty compared to the state-of-the-art is the accurate parameter analysis, conducted with the aim of highlighting which parameters were more significant for valve performance. Finally, the validation results demonstrate that relatively simple and widespread manufacturing techniques [28] can be used to fabricate-in a reliable way and with relatively small deviations from the simulation results-one-way valves that are able to ensure a tight seal-up pressure in the order of $10 \mathrm{kPa}$ magnitude.

\section{Conclusion}

This paper aims at describing the steps needed to design a polymeric valve suitable for application in artificial sphincter systems employed for tackling urinary incontinence diseases. After a preliminary analysis, a commercial valve profile used in the food industry was chosen as reference. By analyzing the commercial valve cross section, nine parameters were extracted, which defined the valve design. Then, a parametric analysis was conducted to identify which factors were more suitable for achieving a significant tuning of the valve performance. This approach allowed us to define a design strategy aimed at minimizing design efforts. After setting the valve diameter, the most influential parameters were valve stiffness and thickness. By varying these parameters, opening pressure tuning in the physiological range was easily achievable.

Appropriate molds and frames for manufacturing and testing polymeric valves were designed and fabricated. Twenty-four poly- meric valves (divided in six different experimental groups) were tested in order to validate the simulations. Experimental Results revealed a maximum error of $23 \%$ compared to simulation predictions with a general good agreement between the envisioned and the actual valve performance. This work will constitute the ground for future advancements on the development of both passive and controllable artificial urinary sphincters.

\section{Acknowledgment}

The authors would like to thank the Fondazione Cassa di Risparmio di Lucca (Lucca, Italy), for providing financial support for this study, in the framework of the SUAVES project (Artificial Urinary System based on bladder and sphincter endoprostheses).

\section{Appendix A. Supplementary data}

Supplementary data associated with this article can be found, in the online version, at http://dx.doi.org/10.1016/j.sna.2015.07.005

\section{References}

[1] D.E. Irwin, Z.S. Kopp, B. Agatep, I. Milsom, P. Abrams, Worldwide prevalence estimates of lower urinary tract symptoms, overactive bladder, urinary incontinence and bladder outlet obstruction, BJU Int. 108 (2011) 1132-1138.

[2] C.R. Bauer, M. Gozzi, W. Hübner, V.W. Nitti, G. Novara, A. Peterson, C.G. Stief, Contemporary management of postprostatectomy incontinence, Eur. Urol. 59 (2011) 985-996.

[3] E. Chung, M. Ranaweera, R. Cartmill, Newer and novel artificial urinary sphincters (AUS): the development of alternatives to the current AUS device, BJU Int. 110 (2012) 5-11.

[4] D.S. Elliott, T.B. Boone, Urethral devices for managing stress urinary incontinence, J. Endourol. 14 (1) (2000) 79-83.

[5] H. Lamraoui, A. Bonvilain, G. Robain, H. Combrisson, S. Basrour, A. Moreau-Gaudry, P. Cinquin, P. Mozer, Development of a novel artificial urinary sphincter: a versatile automated device, IEEE/ASME Trans. Mech. 15 (6) (2010) 916-924.

[6] S. Hached, O. Loutochin, J. Corcos, A. Garon, M. Sawan, Novel, remotely controlled, artificial urinary sphincter: a retro-compatible device, IEEE/ASME Trans. Mech 19 (4) (2014) 1352-1362.

[7] Z. Hached, O. Saadaoui, A. Loutochin, J. Garon, M. Corcos, Novel, wirelessly controlled, and adaptive artificial urinary sphincter, IEEE/ASME Trans Mech. (2015), http://dx.doi.org/10.1109/TMECH.;1; 2015.2389254

[8] N. Pinzi, T., Mazzocchi, G. Giuliani, A structure of artificial endo-urethral sphincter, Patent No. WO2013144770.

[9] H. Mohammadi, K. Mequanint, Prosthetic aortic heart valves: modeling and design, Med. Eng. Phys. 33 (2011) 131-147

[10] G. Burriesci, F.C. Marincola, C. Zervides, Design of a novel polymeric heart valve, J. Med. Eng. Technol. 33 (2010) 7-22.

[11] M.R. Labrosse, C.J. Beller, F. Robicsek, M.J. Thubrikar, Geometric modeling of functional trileaflet aortic valves: development and clinical applications, J. Biomech. 39 (2006) 2665-2672.

[12] A.N. Smuts, et al., Application of finite element analysis to the design of tissue leaflets for a percutaneous aortic valve, J. Mech. Behav. Biomed. Mater. 4 (2011) 85-98.

[13] B. Rahmani, S. Tzamtzis, H. Ghanbari, G. Burriesci, A.M. Seifalian, Manufacturing and hydrodynamic assessment of a novel aortic valve made of a new nanocomposite polymer, J. Biomech. 45 (2012) 1205-1211.

[14] D. Snakenborg, H. Klank, J.P. Kutter, Polymer microvalve with pre-stressed membranes for tunable flow-pressure characteristics, Microfluid. Nanofluid. 10 (2011) 381-388.

[15] R. Mohan, B.R. Schudel, A.V. Desai, J.D. Yearsley, C.A. Apblett, P.J. Kenis, Design considerations for elastomeric normally closed microfluidic valves, Sens. Act. B Chem. 160 (2011) 1216-1223.

[16] A. Jahanshahi, P. Salvo, J. Vanfleteren, PDMS selective bonding for the fabrication of biocompatible all polymer NC microvalves, J. Microelectromech. Syst. 22 (2013) 1354-1360.

[17] W. Hilbert, B. Jakoby, Controlled liquid flow in a microfluidic network with pressure sensitive valves based on polydimethylsiloxane (PDMS)/neodymium (NdFeB) composites, Procedia Eng. 47 (2012) 382-385.

[18] A.I. Hickerson, H.W. Lu, K. Roskos, T. Carey, A. Niemz, Disposable miniature check valve design suitable for scalable manufacturing, Sens. Act. A Phys. 203 (2013) 76-81.

[19] Y. Uohashi, K. Suzumori, H. Taniguchi, Fabrication and evaluation of various types of micro one-way valves through micro rubber molding process, J. Mech. Sci. Technol. 24 (2010) 219-222.

[20] M.M. Elmissiry, A.G. Ali, G.A. Ali, Different urodynamic patterns in female bladder outlet obstruction: can urodynamics alone reach the diagnosis, Arab. J. Urol. 11 (2013) 127-130. 
[21] F. Schmidt, P. Shin, T.M. Jorgensen, J.C. Djurhuus, C.E. Constantinou, Urodynamic patterns of normal male micturition: influence of water consumption on urine production and detrusor function, J. Urol. 168 (2002) $1458-1463$.

[22] W. Schäfer, P. Abrams, L. Liao, A. Mattiasson, F. Pesce, A. Spangberg, P.V. Kerrebroeck, Good urodynamic practices: uroflowmetry, filling cystometry, and pressure-flow studies, Neurourol. Urodynamics 21 (2002) 261-274.

[23] J. Rothschild, L. Chang Kit, L. Seltz, L. Wang, M. Kaufman, R. Dmochowski, D.F. Milam, Difference between urethral circumference and artificial urinary sphincter cuff size, and its effect on postoperative incontinence, J. Urol. 191 (2014) 138-142.

[24] J.C. McDonald, G.M. Whitesides, Poly (dimethylsiloxane) as a material for fabricating microfluidic devices, Acc. Chem. Res. 35 (2002) 491-499.

[25] G.G. Genchi, G. Ciofani, I. Liakos, L. Ricotti, L. Ceseracciu, A. Athanassiou, V. Mattoli, Bio/non-bio interfaces: a straightforward method for obtaining long term PDMS/muscle cell biohybrid constructs, Coll. Surf. B Biointerf. 105 (2013) 144-151.

[26] W.D. Callister, Rethwisch, Materials Science and Engineering: An Introduction, sixth ed., John Wiley \& Sons, Minnesota (USA), 2003, pp. 113-152.

[27] N. Pinzi, T., Mazzocchi, G. Giuliani, A structure of artificial endo-urethral sphincter. WO2013IB52156, 2013.

[28] J.M. Pearce, C.M. Blair, K.J. Laciak, R. Andrews, A. Nosrat, I. Zelenika-Zovko, 3-D printing of open source appropriate technologies for self-directed sustainable development, J. Sustainable Dev. 3 (2010) 17.

\section{Biographies}

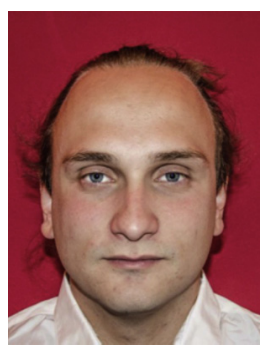

Tommaso Mazzocchi obtained a M.Sc. in Biomedical Engineering in April 2013 at University of Pisa (Italy) with a thesis entitled: "Development and validation of safety systems for magnetically actuated endoluminal robots". He is currently Research Assistant at The BioRobotics Institute of Scuola Superiore Sant'Anna, where he carries out research efforts in the field of biomedical devices, with a particular focus on systems aimed to face urinary incontinence and bladder cancer. He is inventor of 2 international patents related to artificial sphincters and artificial bladders.

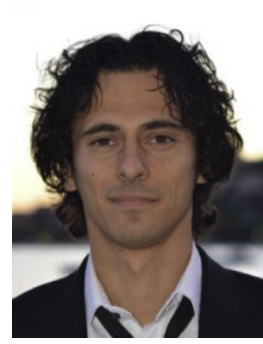

Leonardo Ricotti is Assistant Professor and head of the "Micro-Nano-Bio Systems and Targeted Therapy" Lab at The BioRobotics Institute of Scuola Superiore Sant'Anna. His research interests include biomechatronics, bionics, biomaterials, regenerative medicine and biohybrid systems. He is author of about 50 scientific papers (Source: Scopus), with more than 30 on ISI Journals and 3 book chapters. He is also inventor of 4 patents, related to smart mechatronic devices for medical applications.

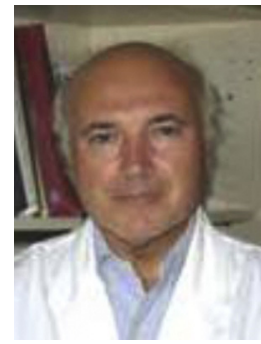

Novello Pinzi obtained a M.Sc. in Medicine and Surgery a the University of Pisa (Italy) in 1976. In 1980 he obtained the specialization in Urology at the Urology Clinic of Florence (Italy), and in 1985 he obtained a second specialization in general surgery at the University of Pisa. Over the past 25 years, he performed a total of 800 interventions. His main fields of interest are bladder reconstruction after radical cystectomy and treatment of urinary incontinence by means of artificial sphincters. He is inventor of 2 international patents related to artificial sphincters and artificial bladders.

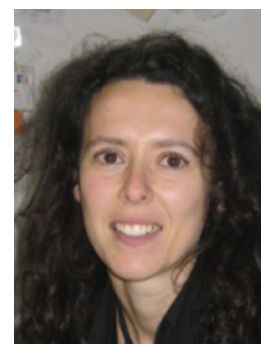

Arianna Menciassi is Full Professor of Biomedica Robotics and leader of the "Surgical Robotics and Allied Technologies" Area, at the BioRobotics Institute of Scuola Superiore Sant'Anna. Her main research interests are in the fields of medical mechatronics, bio-hybrid systems, biomedical micro- and nano-devices and robotic surgery. She possesses an extensive experience in European Projects and international collaborative projects on topics related to robotic and microrobotic diagnosis, surgery and therapy. She is author of about 300 international papers (Source: Scopus), with more than 150 on ISI journals, 1 edited book and 6 book chapters on medical devices and micro-technologies. 